\title{
Asymmetric Synthesis of Highly Functionalized Tetrahydropyrans via a One-Pot Organocatalytic Michael/Henry/Ketalization Sequence
}

\author{
Robert Hahn, Gerhard Raabe and Dieter Enders* \\ Institute of Organic Chemistry, RWTH Aachen University, Landoltweg 1, 52074 Aachen, Germany \\ Supporting Information Placeholder
}

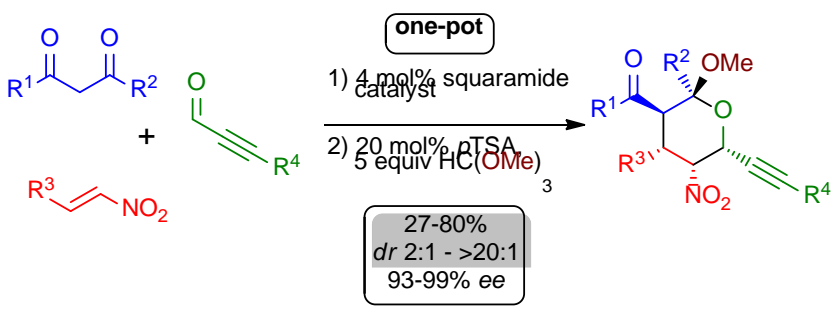

\begin{abstract}
A diastereo- and enantioselective Michael/Henry/ketalization sequence to functionalized tetrahydropyrans is described. The multicomponent cascade reaction uses acetylacetone or $\beta$-keto esters, $\beta$-nitrostyrenes and alkynyl aldehydes as substrates affording tetrahydropyrans with five contiguous stereocenters. Employing a bifunctional quinine-based squaramide organocatalyst, the title compounds are obtained in moderate to good yields (27-80\%), excellent enantiomeric excesses (93-99\% ee) and high diastereomeric ratios $(d r>20: 1)$ after one crystallization.
\end{abstract}

Over the past years we have witnessed a strong increase in the number of publications on organocatalysis as the main topic. $^{1}$ Nowadays numerous groups of organocatalysts are known, with the classes of primary ${ }^{2}$ and secondary amines, ${ }^{3}$ hydrogen-bonding organocatalysts, ${ }^{4}$ chiral phosphoric acids ${ }^{5}$ as well as $N$-heterocyclic carbenes ${ }^{6}$ being used preferentially. The catalytic asymmetric synthesis with these small organic molecules under metal-free conditions now constitutes a rapidly growing research area at the frontier of green chemistry. ${ }^{7}$ Hayashi and co-workers ${ }^{8}$ reported a cross-aldol reaction of alkynyl aldehydes $\mathbf{4}$ with other simple aliphatic aldehydes to obtain synthetically useful $\beta$-alkynyl- $\beta$-hydroxy aldehydes. Similar to the cross-aldol reactions, Henry reactions with $\alpha$ acidic nitro compounds are possible as well. ${ }^{9}$ We wanted to combine these methods by incorporating the resulting alcohol functionality in an intramolecular fashion to generate sixmembered rings. We envisaged the use of alkynyl aldehydes 4 to facilitate the 1,2-addition to be followed up by a ketalization keystep to afford 2-hydroxy tetrahydropyrans, ${ }^{10}$ which to the best of our knowledge would be the first case to generate this type of compounds via a hydrogen-bonding organocatalyst. The triple bond is a versatile structural element that can be used for several transformations, e.g. cycloadditions or selective reductions to alkenes and as a precursor of ketones. The 2hydroxy (or rather alkoxy) tetrahydropyrane unit is a characteristic structural feature of a huge number of natural products, besides carbohydrates for instance of spiroketals, ${ }^{11}$ in soraphen $\mathrm{A}^{12}$ and pederin ${ }^{13}$ as well as in the class of the bryostatins ${ }^{14}$.
To build up the motif of the tetrahydropyran we planned the addition of a $\gamma$-nitro carbonyl compound to an aldehyde. ${ }^{15}$ Achiral $\gamma$-nitro carbonyl compounds were extensively tested but no good asymmetric inductions could be achieved. After intensive literature research, a method provided by Rawal et $a l .{ }^{16}$ was tested. They developed the synthesis of several Michael adducts between 1,3-diketones with $\beta$-nitrostyrenes with a novel squaramide organocatalyst based on cinchonine in excellent yields and enantiomeric excesses. Because of the pseudo-enantiomeric nature of the cinchona alkaloids, we decided to employ a quinine-based organocatalyst to prove the diversity of this method by synthesizing the corresponding enantiomer. According to the protocol of the Rawal group, acetylacetone 1a and $\beta$-nitrostyrene $\mathbf{2 a}$ reacted smoothly with catalyst $\mathbf{A}$ to the Michael adduct $\mathbf{3 a}$ and in the following new step of the one-pot sequence the aldehyde $4 \mathbf{a}$ was added to form the hemiketal 5a (Scheme 1). The product was obtained in an excellent enantiomeric excess of $99 \%$, but in a low yield of $30 \%$ after column chromatography.

Scheme 1. Initial outcome of the envisaged domino sequence 


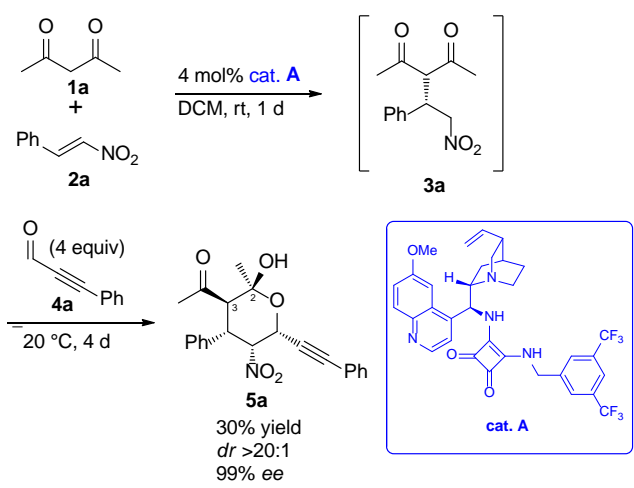

Table 1. Optimizing the reaction temperature for the Henry/ketalization sequence

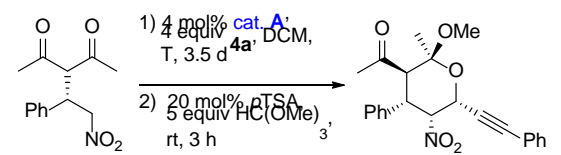

$3 \mathbf{a}$

$6 a$

\begin{tabular}{cccc}
\hline entry $^{a}$ & temp $\left({ }^{\circ} \mathrm{C}\right)$ & yield $(\%)^{b}$ & $\mathrm{dr}^{c}$ \\
1 & $\mathrm{rt}$ & 50 & $3: 1$ \\
2 & 0 & 61 & $8: 1$ \\
3 & -20 & 80 & $>20: 1$ \\
\hline
\end{tabular}

${ }^{a}$ The reaction was performed on a $0.2 \mathrm{mmol}$ scale. ${ }^{b}$ Combined yield of isolated product as a mixture of diastereomers after flash chromatography. 'Diastereomeric ratio; major vs. minor diastereomers determined by ${ }^{1} \mathrm{H}$ NMR.

To investigate this outcome, we stirred the intermediate Michael product 5a over silica gel and found out that a retro-aldol reaction between C2/C3 occurred. Addition of a small amount of base during chromatography to neutralize the acidity of silica resulted in a non-characterizable product. To create a stable compound, we investigated several hydroxyl protecting groups, which all had to react under almost neutral or mild conditions. The best result was obtained with the combination of pTSA and $\mathrm{HC}(\mathrm{OMe})_{3}$ in a quantitative yield and with no loss of enantioselectivity. With this knowledge in hand we added the protecting reagents to the reaction mixture and thus we were able to get the desired product in a good yield. Knowing how to overcome the stability problems, we screened for the best reaction conditions. A short temperature screening was conducted with Michael adduct 3a and aldehyde 4a (Table 1). Reducing the reaction temperature from room temperature to $-20{ }^{\circ} \mathrm{C}$ (entries $1-3$ ) was followed by an increase in yield and diastereoselectivity. Now we focused on the one-pot procedure for the synthesis of the tetrahydropyrans. Following the protocol of the Rawal group, we started with the addition of acetylacetone (1a) and $\beta$-nitrostyrene (2a) with $4 \mathrm{~mol} \%$ of catalyst in DCM at room temperature (Table 2, entry 1). As opposed to the 2 equivalents of acetylacetone of the Rawal group, we used a 1:1 ratio, because excess acetylacetone would react with aldehyde $\mathbf{4 a}$ in an aldol-condensation and thus increase the complexity of the final mixture. Reducing the amount of catalyst was not successful. Although the first step occured quantitatively, we could obtain only 53\% yield at $2 \mathrm{~mol} \%$ catalyst loading, respectively $11 \%$ yield at 0.7 mol\% (entries 2 and 3 ). For the next set of modifications we changed the amount of aldehyde 4a (Table 2, entry 4-7). With 10 equivalents a lower yield as compared to 4 or 2 equivalents was obtained indicating a concentration issue. Further reduction of the amount of the aldehyde led to a small decrease in yield (entries 6 and 7). Extending the reaction time resulted in no increase in yield (entries 8-10). The amount of solvent was reduced to a concentration of $0.5 \mathrm{M}$, which resulted in an increase of yield (Table 2, entry 11). Having the proper conditions in hand, an extension of the scope was investigated (Table 3). Several different substituents $\mathrm{R}^{3}$ on the aryl moiety of $\mathbf{2}$ (entries $\mathbf{b}-\mathbf{d}, \mathbf{f}$ ) were introduced giving moderate to good yields of $27-65 \%$ and very good enantioselectivities (93-97\% ee). Even a heterocyclic group, like the protected $N$-Boc-indolyl, could be used (Table 3, entry e).

Table 2. Screening for the optimal conditions
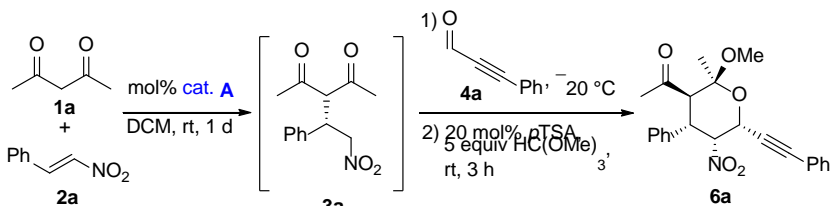

$2 a$

3a

\begin{tabular}{cccccc}
\hline entry $^{a}$ & mol\% & equiv of 3 & ${\text { time }(\mathrm{d})^{b}}^{\text {yield }_{(\%)^{c}}}$ & $\mathrm{dr}^{d}$ \\
1 & 4 & 4 & 4 & 62 & $>20: 1$ \\
2 & 2 & 4 & 5 & 53 & $>20: 1$ \\
3 & 0.7 & 4 & 5 & 11 & n.d. ${ }^{e}$ \\
4 & 4 & 10 & 5 & 46 & $>20: 1$ \\
5 & 4 & 2 & 5 & 61 & $>20: 1$ \\
6 & 4 & 1.5 & 5 & 52 & $>20: 1$ \\
7 & 4 & 1.1 & 5 & 50 & $>20: 1$ \\
8 & 4 & 2 & 6 & 59 & $>20: 1$ \\
9 & 4 & 2 & 7 & 57 & $>20: 1$ \\
10 & 4 & 2 & 9 & 59 & $>20: 1$ \\
$11^{f}$ & 4 & 2 & 5.5 & 79 & $>20: 1$ \\
\hline
\end{tabular}

${ }^{a}$ The reaction was performed on a $0.2 \mathrm{mmol}$ scale $(0.2 \mathrm{M}$ in DCM). ${ }^{b}$ Sum of reaction time. ${ }^{c}$ Combined yield of isolated product as a mixture of diastereomers after flash chromatography. ${ }^{d}$ Diastereomeric ratio: major vs. minor diastereomers determined by ${ }^{1} \mathrm{H}$ NMR. ${ }^{e}$ Not determined. ${ }^{f}$ Conducted in $0.4 \mathrm{~mL}$ of solvent (0.5 M).

The aromatic part $\mathrm{R}^{4}$ of the aldehyde 4 was substituted with electron-donating and electron-withdrawing groups to yield the cascade product in modest to good yields (61-80\%) and in very good enantioselectivities of 94-97\% ee (Table 3, entries $\mathbf{g}-\mathbf{i}$ ). Switching to a cyclopentyl moiety (entry j) led to the same range of yield (68\%) and enantioselectivity (96\% ee). Desym- 
metrization of the acetylacetone to the corresponding methyl ester gave $60 \%$ yield and $97 \%$ ee (Table 3, entry k). Increasing the bulkiness to a tert-butoxy group (entry $\mathbf{m}$ ) resulted in a drop of obtained product (34\% yield) but still impressive 98\% ee. A further domino product was obtained in $69 \%$ yield and $96 \%$ ee after extending the side chain to an ethyl group (Table 3 , entry l).

The relative configuration was determined by NOE measurements for compound 6c (Figure 1) as well as the absolute configuration by single crystal X-ray analysis of compound $\mathbf{6 a}$ (Figure 2). ${ }^{17}$

Figure 1. Determination of the relative configuration by NOE for compound $\mathbf{6 c}$.

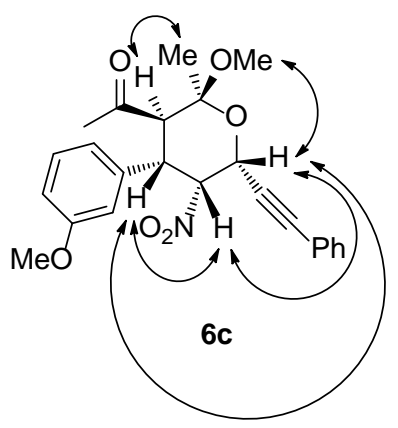


Table 3. Scope of the Michael/Henry/ketalization sequence to form tetrahydropyrans

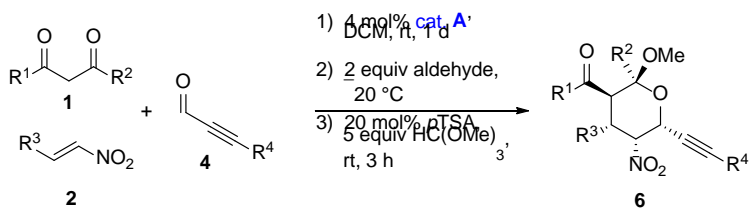

\begin{tabular}{|c|c|c|c|c|c|c|c|c|}
\hline $\mathbf{6}^{a}$ & $\mathrm{R}^{1}$ & $\mathrm{R}^{2}$ & $\mathrm{R}^{3}$ & $\mathrm{R}^{4}$ & time $(\mathrm{d})^{b}$ & yield $(\%)^{c}$ & $\mathrm{dr}^{d}$ & $\overline{\text { ee }(\%)^{e}}$ \\
\hline $\mathbf{a}$ & $\mathrm{Me}$ & $\mathrm{Me}$ & $\mathrm{Ph}$ & $\mathrm{Ph}$ & 5.5 & 79 & $>20: 1$ & $>99$ \\
\hline b & $\mathrm{Me}$ & $\mathrm{Me}$ & $2-\mathrm{BrC}_{6} \mathrm{H}_{4}$ & $\mathrm{Ph}$ & 5.5 & 65 & $13: 1$ & 93 (99) \\
\hline c & $\mathrm{Me}$ & $\mathrm{Me}$ & 3- $\mathrm{MeOC}_{6} \mathrm{H}_{4}$ & $\mathrm{Ph}$ & 6.5 & 45 & $6: 1$ & 97 (99) \\
\hline d & $\mathrm{Me}$ & $\mathrm{Me}$ & $3,4-\mathrm{OCH}_{2} \mathrm{OC}_{6} \mathrm{H}_{3}$ & $\mathrm{Ph}$ & 5 & 27 & $2: 1$ & 95 (99) \\
\hline e & $\mathrm{Me}$ & $\mathrm{Me}$ & 3-(N-Boc-indolyl) & $\mathrm{Ph}$ & 6 & 38 & $8: 1$ & 95 \\
\hline $\mathbf{f}$ & $\mathrm{Me}$ & $\mathrm{Me}$ & $4-\mathrm{NO}_{2} \mathrm{C}_{6} \mathrm{H}_{4}$ & $\mathrm{Ph}$ & 9 & 46 & $2: 1$ & 93 \\
\hline g & $\mathrm{Me}$ & $\mathrm{Me}$ & $\mathrm{Ph}$ & $3-\mathrm{FC}_{6} \mathrm{H}_{4}$ & 5.5 & 67 & $5: 1$ & 94 (99) \\
\hline $\mathbf{h}$ & $\mathrm{Me}$ & $\mathrm{Me}$ & $\mathrm{Ph}$ & $4-\mathrm{MeC}_{6} \mathrm{H}_{4}$ & 5.5 & 61 & $7: 1$ & 97 \\
\hline i & $\mathrm{Me}$ & $\mathrm{Me}$ & $\mathrm{Ph}$ & $2-\mathrm{MeOC}_{6} \mathrm{H}_{4}$ & 5.5 & 80 & $3: 1$ & 95 (99) \\
\hline $\mathbf{j}$ & $\mathrm{Me}$ & $\mathrm{Me}$ & $\mathrm{Ph}$ & cyclopentyl & 5.5 & 68 & $4: 1$ & 96 \\
\hline $\mathbf{k}$ & OMe & $\mathrm{Me}$ & $\mathrm{Ph}$ & $\mathrm{Ph}$ & 5.5 & 60 & $4: 1$ & 97 (99) \\
\hline 1 & $\mathrm{OMe}$ & Et & $\mathrm{Ph}$ & $\mathrm{Ph}$ & 5 & 69 & $3: 1$ & 96 (99) \\
\hline m & $\mathrm{O}^{t} \mathrm{Bu}$ & $\mathrm{Me}$ & $\mathrm{Ph}$ & $\mathrm{Ph}$ & 9 & 34 & $2: 1$ & 98 \\
\hline
\end{tabular}

${ }^{a}$ The reaction was performed on a $0.4 \mathrm{mmol}$ scale $\left(0.5 \mathrm{M}\right.$ in DCM). ${ }^{b}$ Sum of reaction time. ${ }^{c}$ Combined yield of isolated product as a mixture of diastereomers after flash chromatography. ${ }^{d}$ Diastereomeric ratio: major vs. minor diastereomers determined by ${ }^{1} \mathrm{H}$ NMR; after one recrystallization $d r>20: 1$. ${ }^{e}$ Determined by HPLC analysis on a chiral stationary phase for the major diastereomer; value in bracket after one recrystallization.

In summary, we have developed an organocatalytic Michael/Henry/ketalization cascade sequence to access highly funtionalized tetrahydropyrans. A hydrogen-bonding organocatalyst on a squaramide basis was used to merge acetylacetone or different $\beta$-keto esters with nitroalkenes and ynals. In this manner tetrahydropyrans, bearing five contiguous stereocenters were obtained in moderate to good yields (27-80\%), after one recrystallization in high diastereomeric ratios $(d r$ $>20: 1)$ and excellent enantiomeric excesses (93-99\% ee).

Figure 2. Determination of the absolute configuration by X-ray crystal structure analysis of compound $\mathbf{6 a}$.

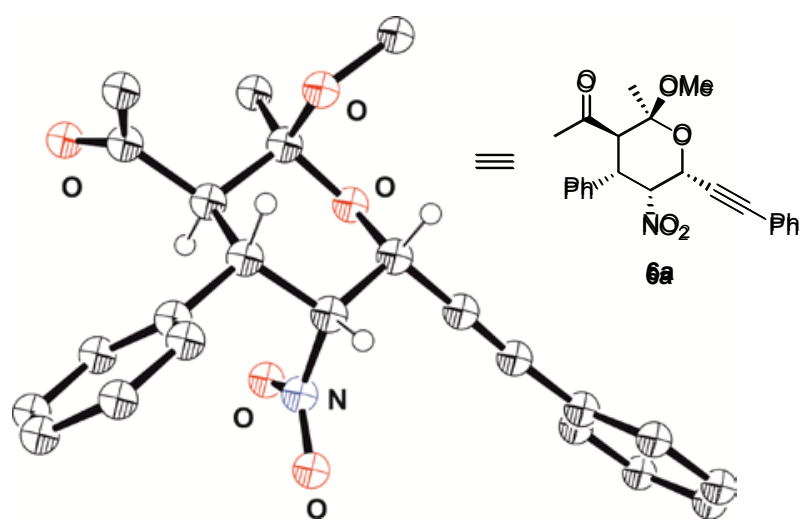

\section{ASSOCIATED CONTENT}

\section{Supporting Information}

Experimental procedures and the characterization of all products are reported in the supporting information. This material is available free of charge via the Internet at http://pubs.acs.org.

\section{AUTHOR INFORMATION}

\section{Corresponding Author}

*E-mail: enders@rwth-aachen.de; Fax: (+49)-241-809-2127.

Notes

The authors declare no competing financial interest.

\section{ACKNOWLEDGMENT}

Support from the European Research Council (ERC Advanced Grant "DOMINOCAT") is gratefully acknowledged.

\section{REFERENCES}

(1) For selected reviews, see: (a) Enders, D.; Grondal, C.; Hüttl, M. R. M. Angew. Chem. 2007, 119, 1590; Angew. Chem. Int. Ed. 2007, 46, 1570. (b) Walji, A. M.; MacMillan, D. W. C. Synlett 2007, 1477. (c) Grondal, C.; Jeanty, M.; Enders, D. Nat. Chem. 2010, 2, 167. (d) Pellissier, H. Adv. Synth. Catal. 2012, 354, 237. (e) Lu, L.-Q.; Chen, J.-R.; Xiao, W.-J. Acc. Chem. Res. 2012, 45, 1278. (f) Goudedranche, S.; Raimondi, W.; Bugaut, X.; Constantieux, T.; Bonne, D.; Rodriguez, J. Synthesis 2013, 45, 1909. (g) Volla, C. M. R.; Atodiresei, I.; Rueping, M. Chem. Rev. 2014, 114, 2390.

(2) For selected reviews and examples on cinchona alkaloidderived primary amine catalysis, see: (a) Xu, L.-W.; Luo, J.; Lu, Y. 
Chem. Commun. 2009, 1807. (b) Melchiorre, P. Angew. Chem. Int. Ed. 2012, 51, 9748; Angew. Chem. 2012, 51, 9889. (c) Cassani, C.; Martín-Rapún, R.; Arceo, E.; Bravo, F.; Melchiorre, P. Nat. Protoc. 2013, 8, 325. (d) Hack, D.; Enders, D. Synthesis 2013, 45, 2904. (e) Moran, A.; Hamilton, A.; Bo, C.; Melchiorre, P. J. Am. Chem. Soc. 2013, 135, 9091. (f) Kang, Y. K.; Kim, D. Y. Adv. Synth. Catal. 2013, 355, 3131. (g) Duan, J.; Li, P. Catal. Sci. Technol. 2014, 4, 311.

(3) For selected examples on secondary amine organocatalysts, see: (a) Rueping, M.; Kuenkel, A.; Tato, F.; Bats, J. W. Angew. Chem. Int. Ed. 2009, 48, 3699. (b) Rueping, M.; Haack, K.; Ieasuwan, W.; Sundén, H.; Blanco, M.; Schoepke, F. R. Chem. Commun. 2011, 47, 3828. (c) Enders, D.; Greb, A.; Deckers, K.; Selig, P.; Merkens, C. Chem. Eur. J. 2012, 18, 10226. (d) Enders, D.; Joie, C.; Deckers, K. Chem. Eur. J. 2013, 19, 10818. (e) Chatterjee, I.; Bastida, D.; Melchiorre, P. Adv. Synth. Catal. 2013, 355, 3124. (f) Zeng, X.; Ni, Q.; Raabe, G.; Enders, D. Angew. Chem. Int. Ed. 2013, 52, 2977. (g) Erdmann, N.; Philipps, A. R.; Atodiresei, I.; Enders, D. Adv. Synth. Catal. 2013, 355, 847. (h) Dong, L.-J.; Fan, T.-T.; Wang, C.; Sun, J. Org. Lett. 2013, 15, 204. (i) Wu, L.; Wang, Y.; Song, H.; Tang, L.; Zhou, Z.; Tang, C. Chem. Asian J. 2013, 8, 2204. (j) Alexakis, A.; Lefranc, A.; Guénée, L. Org. Lett. 2013, 15, 2172. (k) Joie, C.; Deckers, K.; Enders, D. Synthesis 2014, 46, 799.

(4) For selected reviews and examples of hydrogenbonding catalysis, see: (a) Schreiner, P. R. Chem. Soc. Rev. 2003, 32, 289. (b) Taylor, M. S.; Jacobsen, E. N. Angew. Chem. 2006, 118, 1550; Angew. Chem. Int. Ed. 2006, 45, 1520. (c) Connon, S. J. Chem. Eur. J. 2006, 12, 5418. (d) Doyle, A. G.; Jacobsen, E. N. Chem. Rev. 2007, 36, 5713. (e) Zhang, Z.; Schreiner, P. R. Chem. Soc. Rev. 2009, 38, 1187. (f) Etzenbach-Effers, K.; Berkessel, A. Top. Curr. Chem. 2009, 291, 1. (g) Aleman, J.; Parra, A.; Jiang, H.; Jørgensen, K. A. Chem. Eur. J. 2011, 17, 6890. (h) Storer, R. I.; Aciro, C.; Jones, L. H. Chem. Soc. Rev. 2011, 40, 2330. (i) Albrecht, Ł.; Dickmeiss, G.; Acosta, F. C.; Rodriguez-Escrich, C.; Davis, R. L.; Jørgensen, K. A. J. Am. Chem. Soc. 2012, 134, 2543. (j) Enders, D.; Urbanietz, G.; CassensSasse, E.; Kees, S.; Raabe, G. Adv. Synth. Catal. 2012, 354, 1481. (k) Loh, C. C. J.; Hack, D.; Enders, D. Chem. Commun. 2013, 49, 10230. (l) Liu, Y.; Wang, Y.; Song, H.; Zhou, Z.; Tang, C. Adv. Synth. Catal. 2013, 355, 2544. (m) Gosh, A. K.; Zhou, B. Tetrahedron Lett. 2013, 54, 3500. (n) Sun, W.; Hong, L.; Zhu, G.; Wang, Z.; Wie, X.; Ni, J.; Wang, R. Org. Lett. 2014, 16, 544. (o) Zhou, E.; Liu, B.; Dong, C. Tetrahedron: Asymmetry 2014, 25, 181. (p) Han, X.; Dong, C.; Zhou, H.-B. Adv. Synth. Catal. 2014, 356, 1275.

(5) (a) Akiyama, T.; Itoh, J.; Yokota, K.; Fuchibe, K. Angew. Chem. 2004, 116, 1592; Angew. Chem. Int. Ed. 2004, 43, 1566. (b) Uraguchi, D.; Terada, M. J. Am. Chem. Soc. 2004, 126, 5356. (c) Rueping, M.; Azap, C. Angew. Chem. Int. Ed. 2006, 45, 7832. (d) Baudequin, C.; Zamfir, A.; Tsogoeva, S. B. Chem. Commun. 2008, 4637. (e) Evans, C. G.; Gestwicki, J. E. Org. Lett. 2009, 11, 2957. (f) Terada, M. Synthesis, 2010, 1929. (g) Taylor, J. E.; Daniels, D. S. B.; Smith, A. D. Org. Lett. 2013, 15, 6058. (h) Courant, T.; Kumarn, S.; He, L.; Retailleau, P.; Masson, G. Adv. Synth. Catal. 2013, 355, 836. (i) Izquierdo, J.; Orue, A.; Scheidt, K. A. J. Am. Chem. Soc. 2013, 135, 10634. (j) Enders, D.; Stöckel, B. A.; Rembiak, A. Chem. Commun. 2014, 50, 4489. (k) Tian, X.; Hofmann, N.; Melchiorre, P. Angew. Chem. Int. Ed. 2014, 53, 2997. (l) Mori, K.; Wakazawa, M.; Akiyama, T. Chem. Sci. 2014, 5, 1799. (m) Zhong, S.; Nieger, M.; Bihlmeier, A.; Shi, M.; Bräse, S. Org. Biomol. Chem. 2014, 12, 3265.

(6) For selected reviews and examples on NHC catalysis, see: (a) Heitbaum, M.; Glorius, F.; Escher, I. Angew. Chem. Int. Ed. 2006, 45, 4732. (b) Enders, D.; Niemeier, O.; Henseler, A. Chem. Rev. 2007, 107, 5606. (c) Moore, J. L.; Rovis, T. Top. Curr. Chem. 2010, 291, 118. (d) Biju, A. K.; Kuhl, N.; Glorius, F. Acc. Chem. Res. 2011, 44, 1182. (e) Grossmann, A.; Enders, D. Angew. Chem. 2012, 124, 320; Angew. Chem. Int. Ed. 2012, 51, 314. (f) Izquierdo, J.; Hutson, G. E.; Cohen, D. T.; Scheidt, K. A. Angew. Chem. 2012, 124, 11854; Angew. Chem. Int. Ed. 2012, 51, 11686. (g) Bugaut, X.; Glorius, F. Chem. Soc. Rev. 2012, 41, 3511. (h) Ni, Q.; Zhang, H.; Grossmann, A.; Loh, C. C. J.; Merkens, C.; Enders, D. Angew. Chem. Int. Ed. 2013, 52, 13562. (i) Ryan, S. J.; Candish, L.; Lupton, D. W.; Chem. Soc. Rev. 2013, 42, 4906. (j) Song, X.; Ni, Q.; Grossmann, A.;
Enders, D. Chem. Asian J. 2013, 8, 2965. (k) P. Chauhan, D. Enders, Angew. Chem. Int. Ed. 2014, 53, 1485.

(7) (a) Wende, R. C.; Schreiner, P. R. Green Chem. 2012, 14, 1821. (b) Liu, D. D. J.; Chen, E. Y.-X. Green Chem. 2014, 16, 964.

(8) Hayashi, Y.; Kojima, M.; Yasui, Y.; Kanda, Y.; Mukaiyama, T.; Shomura, H.; Nakamura, D.; Ritmaleni, Sato, I. ChemCatChem 2013, 5, 2887.

(9) For recent selected examples of organocatalytic Michael-Henry domino reactions, see: (a) Xu, D.-Q.; Wang, Y.-F.; Luo, S.-P.; Zhang, S.; Zhong, A.-G.; Chen, H.; Xu, Z.-Y. Adv. Synth. Catal. 2008, 350, 2610. (b) Tan, B.; Chua, P. J.; Li, Y.; Zhong, G.Org. Lett. 2008, 10, 2437. (c) Tsakos, M.; Elsegood, M. R. J.; Kokotos, C. G. Chem. Commun. 2013, 49, 2219. (d) Qian, H.; Zhao, W.; Sung, H. H.-Y.; Williams, I. D.; Sun, J. Chem. Commun. 2013, 49, 4361. (e) Quintavalla, A.; Lombardo, M.; Sanap, S. P.; Trombini, C. Adv. Synth. Catal. 2013, 355, 938. (f) Dai, Q.; Arman, H.; Zhao, J. C.-G. Chem. Eur. J. 2013, 19, 1666. (g) Arai, T.; Yamamoto, Y. Org. Lett. 2014, 16, 1700 .

(10) For selected recent examples on tetrahydropyran syntheses, see: (a) Heumann, L. V.; Keck, G. E. Org. Lett. 2007, 9, 1951. (b) Larrosa, I.; Romea, P.; Urpi, F. Tetrahedron 2008, 64, 2683. (c) Gotoh, H.; Okamura, D.; Ishikawa, H.; Hayashi, Y. Org. Lett. 2009, 11, 4056. (d) Chandrasekhar, S.; Mallikarjun, K.; Pavankumarreddy, G.; Rao, K. V.; Jagadeesh, B. Chem. Commun. 2009, 45, 4985. (e) Olier, C.; Kaafarani, M.; Gastaldi, S.; Bertrand, M. P. Tetrahedron 2010, 66, 413. (f) Urbanietz, G.; Atodiresei, I.; Enders, D. Synthesis 2014, 46, 1261.

(11) (a) Perron, F.; Albizati, K. F. Chem. Rev. 1989, 89, 1617. (b) Raju, B. R.; Saikia, A. K. Molecules 2008, 13, 1942. (c) Favre, S.; Vogel, P.; Gerber-Lemaire, S. Molecules 2008, 13, 2570. (d) Sperry, J.; Liu, Y.-C.; Brimble, M. A. Org. Biomol. Chem. 2010, 8, 29.

(12) Bedorf, N.; Schomburg, D.; Gerth, K.; Reichenbach, H.; Höfle, G. Liebigs Ann. Chem. 1993, 1017.

(13) (a) Willson, T. M.; Kocienski, P.; Jarowicki, K.; Isaac, K.; Faller, A.; Campbell, S.; Bordner, J. Tetrahedron 1990, 46, 1757. (b) Willson, T. M.; Kocienski, P.; Jarowicki, K.; Isaac, K.; Faller, A.; Campbell, S.; Bordner, J. Tetrahedron 1990, 46, 1767.

(14) Hale, K. J.; Hummersone, M. G.; Manaviazar, S.; Frigerio, M. Nat. Prod. Rep. 2002, 19, 413.

(15) Enders, D.; Hahn, R.; Atodiresei, I. Adv. Synth. Catal. 2013, 355, 1126.

(16) Malerich, J. P.; Hagihara, K.; Rawal, V. H. J. Am. Chem. Soc. 2008, 130, 14416.

(17) CCDC 999310 (6a) contains the supplementary crystallographic data for this paper. These data can be obtained free of charge from The Cambridge Crystallographic Data Centre via www.ccdc.cam.ac.uk/data_request/cif. 
For Table of Contents Only

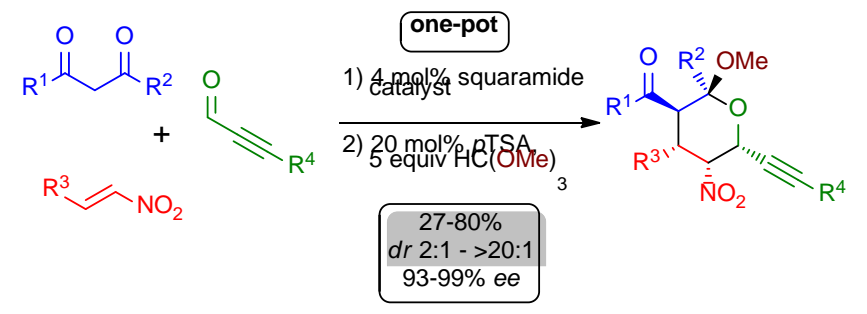

